\title{
Optical parameters relevant for High Angular Resolution at Paranal from GSM instrument and surface layer contribution
}

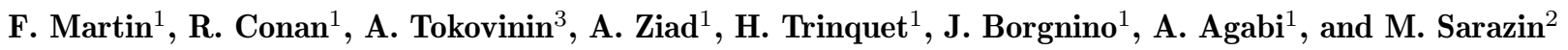 \\ 1 U.M.R. Astrophysique No. 6525, Université de Nice-Sophia Antipolis, F-06108 Nice Cedex 2, France \\ 2 European Southern Observatory, Karl-Schwarzschild-Str. 2, D-85748 Garching bei München, Germany \\ 3 Sternberg Astronomical Institute, Universitestsky prosp. 13, 119899 Moscow, Russia
}

Received January 12; accepted February 25, 2000

\begin{abstract}
Main atmospheric parameters characterizing the turbulence were measured at the Paranal ESO Observatory (Chile) with the Generalized Seeing Monitor (GSM) during 19 nights in November-December 1998. The median seeing for this period was $0.88^{\prime \prime}$, a little worse than average, the spatial coherence outer scale of the wavefront $\mathcal{L}_{0}=22.0 \mathrm{~m}$ and the median isoplanatic angle $\theta_{0}=1.90^{\prime \prime}$. A very good agreement with seeing values given by ESO DIMM was found. Effects of ground layer turbulence were estimated from measurements of temperature micro-fluctuations at several altitudes. These measurements revealed that the first $21 \mathrm{~m}$ above ground account for $12.5 \%$ of the total turbulent energy. Comparison between GSM and ESO DIMM values have permitted to highlight a local phenomenon which occurs sometimes at the site in the middle of the night. Statistics of the refractive index structure constant $C_{n}^{2}$ in the surface layer is also studied.
\end{abstract}

Key words: turbulence — atmospheric effects — site testing

\section{Introduction}

It is well known that performances of high angular resolution techniques (HAR) are severely limited by the random optical effects induced by the turbulent atmosphere on the light propagation. A quantitative spatial and temporal characterization of the corrugated wavefront at the ground is necessary to optimize the operation of adaptive optics (AO) and long baseline interferometry (LBI) instruments and to guide the future development of these techniques.

Send offprint requests to: F. Martin, e-mail: Francois.MARTIN@unice.fr
The parameters which characterize the wavefront statistics from an optical point of view are the well known Fried's parameter $r_{0}(\lambda)$, the spatial coherence outer scale of the wavefront $\mathcal{L}_{0}$ (called outer scale hereafter), which becomes of importance when comparable to the telescope aperture, the isoplanatic angle $\theta_{0}(\lambda)$, and the characteristic time which determines the response time for the deformable mirror in $\mathrm{AO}$ or for the fringe tracking in LBI.

A specific instrument named GSM (Generalized Seeing Monitor) dedicated to the monitoring of these parameters has been built. It has been essentially developed (Martin et al. 1994) to evaluate the outer scale statistics because no systematic measurements existed for it. The scattered values available in the literature were deduced from various techniques and were somewhat controversial (Mariotti et al. 1984; Colavita et al. 1987; Rigaut et al. 1991; Ziad et al. 1994; Buscher et al. 1995; Takato et al. 1995; Davis et al. 1995; Beriot et al. 1997).

The VLT system with its four $8 \mathrm{~m}$ apertures is a set of optical telescopes. It is located at the ESO Cerro Paranal observatory in Chile which is among the major world astronomical sites. The properties of the atmospheric turbulence at this site are of the utmost importance for the operation of this unique system.

A statistics of seeing and cloud coverage at Paranal over several years was obtained from the Differential Image Motion Monitor of ESO (Sarazin \& Roddier 1990) and shows it to be one of the lowest among those measured at several astronomical sites, with the long-term median seeing of $0.70^{\prime \prime}$. However, the information on some other properties of atmosphere at Paranal was missing. The aim of our measurement campaign with GSM in December 1998 was to provide statistics of the outer scale $\mathcal{L}_{0}$ and of the isoplanatic angle $\theta_{0}$. The corresponding results are presented in Sect. 3.

Previously, the GSM worked successfully at the ESO La Silla observatory (Martin et al. 1998b), and in 


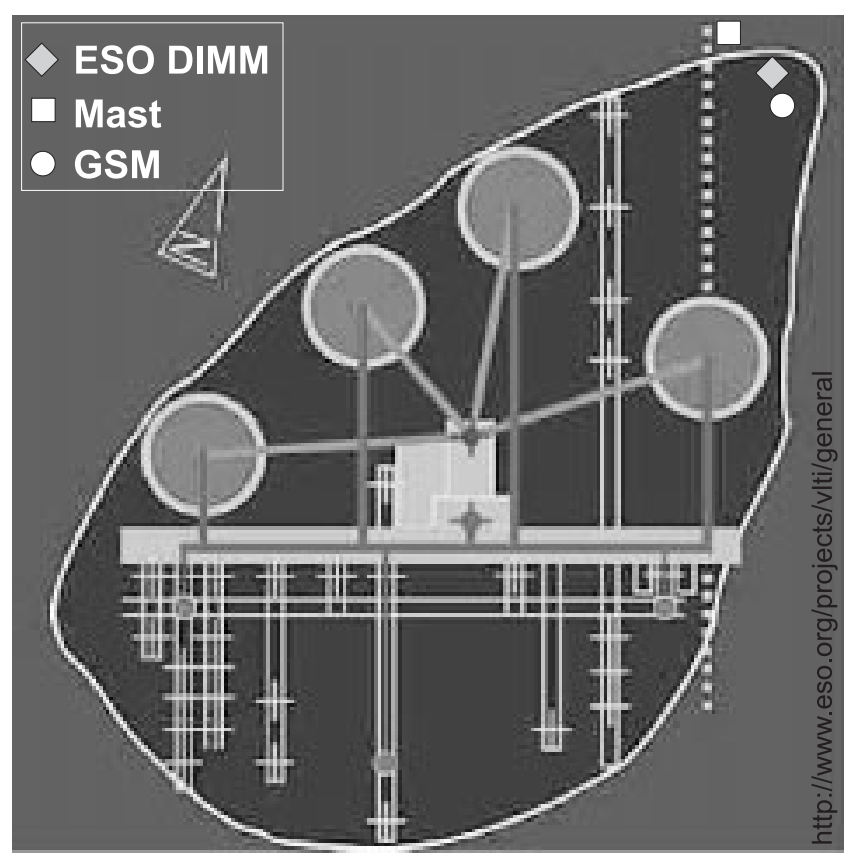

Fig. 1. The plan of Paranal site and the location of the equipment

some other observatories over the world (Oukaïmeden in Morocco, Maydanak in Uzbekistan, and Cerro Pachon in Chile, the southern site for the Gemini project).

The schematic plan of the Paranal site is given in Fig. 1. The pillars with GSM telescopes were located near the ESO DIMM seeing monitor. A pre-existing mast was used to install three couples of temperature sensors for temperature micro-fluctuation measurements in the Surface Layer (SL).

All atmospheric parameters given in this report are calculated for a wavelength $\lambda=500 \mathrm{~nm}$ for observations at zenith. Seeing angle $\varepsilon$ is the full width at half maximum (FWHM) of a long-exposure stellar image in a large telescope, $\varepsilon=0.98 \lambda / r_{0}$. Outer scale $\mathcal{L}_{0}$ is a $C_{n}^{2}$-weighted average of the local turbulence outer scale $\mathcal{L}_{0}(\mathrm{~h})$, as defined by Borgnino (1990). The isoplanatic angle $\theta_{0}$ is relevant for adaptive optics (Fried 1982).

\section{Principle of measurements}

Although the Generalized Seeing Monitor was the main instrument used in this campaign, we devote little space to its description, which can be found in our previous works.

\subsection{Principle of the GSM}

In its initial version GSM is presented in Martin et al. (1994). The actual instrument is described in Martin et al. (1998b) and Tokovinin et al. (1998b). Description and performance analysis are also given in Ziad et al. (1999). We refer the interested readers to the reports on the previous GSM missions (Martin et al. 1998a and Tokovinin et al. 1998a).

The GSM instrument consists of four 10-cm telescopes with their individual image analysis devices to sense the angle of arrival (AA) fluctuations in one direction, called modules. The telescopes are installed on three equatorial mounts; two modules share the same mount and are working as the ESO DIMM. At a distance of $1 \mathrm{~m}$ to the south of these modules a third one is installed on a separate mount. The fourth one, with its mount, is located at $0.8 \mathrm{~m}$ to the east. The same L-shaped configuration has been used in the previous GSM missions except at Maydanak site.

GSM modules and their mounts were installed on top of 3 concrete pillars, with the height of telescopes $1.7 \mathrm{~m}$ above ground. From 3 sides the experiment was surrounded by a wind-protective enclosure consisting of a $2 \mathrm{~m}$ high net.

Atmospheric AA fluctuations are measured by each module in the declination direction, with $5 \mathrm{~ms}$ time resolution. A typical measurement consists of a $2 \mathrm{mn}$ long data acquisition. It is processed immediately, providing the estimate of the Fried coherence diameter $r_{0}$ (from differential AA fluctuations in modules 1 and 2), outer scale $\mathcal{L}_{0}$ (inferred from the covariances of $\mathrm{AA}$ and computed via the Von Karman model for the phase spectrum of the turbulent atmosphere), and isoplanatic angle $\theta_{0}$ (from scintillation index).

The ESO DIMM seeing monitor (Sarazin \& Roddier 1990) which operates at the Paranal site continuously was located at a distance of the order of $10 \mathrm{~m}$ from GSM. It is installed on a $6 \mathrm{~m}$ high tower, in order to avoid the near-ground turbulence effects as much as possible.

\subsection{Temperature micro-fluctuation measurements in the surface layer}

Turbulence sensing in the SL has been undertaken in order to gain a better understanding of the turbulence effects near the ground. For balloon measurements of the vertical turbulence profiles, very sensitive temperature sensors have been developed in our laboratory. Each probe consists of two thin-wire sensors mounted at a separation of $1 \mathrm{~m}$ on a rod. The structure constant of temperature fluctuations, $C_{\mathrm{T}}^{2}$, is calculated from the dispersion of the temperature difference between the pair of sensors. The time resolution of the micro-thermometers with their electronics and transmitting devices is about $5 \mathrm{~ms}$ and an integrated value of the structure constant is transmitted to the ground each $1.5 \mathrm{~s}$. The typical noise level can reach a few $10^{-8}$ (Celsius degree) ${ }^{2}$. The refractive index structure constant $C_{n}^{2}$ is calculated from $C_{\mathrm{T}}^{2}$ using the appropriate values of mean temperature and pressure. $C_{n}^{2}$ values are obtained with a precision around $10^{-20} \mathrm{~m}^{-2 / 3}$. Previous 
results obtained with this equipment can be found in Vernin \& Muñoz-Tuñon (1992).

Four pairs of sensors were installed on the meteorological mast at the heights of $3,7,21$, and $31 \mathrm{~m}$ above the base of the mast. The upper sensor, however, was damaged, and only the remaining 3 have provided valid data. It must be noted that the base of the mast was somewhat lower (by $1 \mathrm{~m}$ ) than the ground level of GSM/DIMM location. Hence the sensors at 3 and $7 \mathrm{~m}$ are at the same heights as GSM and DIMM, respectively.

\section{The GSM campaign at Cerro Paranal Observatory}

The GSM instrument has been transported to Paranal after the completion of the previous mission in October 1998 at Cerro Pachon (Chile).

The excellent quality of Paranal site has permitted to collect 1884 acquisitions during 19 nights, more than in any previous GSM campaign.

Micro-thermal data acquisitions have been operational only during 9 nights.

\subsection{Results of the campaign}

Throughout this report the universal time (UT) is given. A date always refers to the beginning of the night. The data summary for the mission is presented in Table 1 . In the first columns we give date, median speed and direction of the wind at $10 \mathrm{~m}$ altitude. Next 3 columns contain the nightly median values of main atmospheric parameters measured by GSM, namely seeing $\varepsilon$, outer scale $\mathcal{L}_{0}$ and isoplanatic angle $\theta_{0}$. In the last column the seeing values computed by the ESO DIMM are listed.

During our mission the overall median seeing was slightly worse than long-term average for Paranal $\left(0.70^{\prime \prime}\right)$, but typical for the whole 1998 year, with its monthly average seeing of $0.9^{\prime \prime}-1^{\prime \prime}$. We note a very good stability of atmospheric parameters. Notably, in the period of December $1-5$ surprisingly little variation of all main parameters occurred. Similar values were found again on December 15,16 and 17 .

The graphic representation of the ensemble of GSM data is given in Fig. 2 .

\subsection{Outer scale statistics}

GSM is the first instrument capable to measure the outer scale $\mathcal{L}_{0}$ continuously and to study the statistics of this important parameter. During its previous missions it was found that $\mathcal{L}_{0}$ follows a log-normal distribution with a dispersion of $\log \left(\mathcal{L}_{0}\right)$ of about 0.23 , and, rather similar median values, ranging from 24 to $31 \mathrm{~m}$, were obtained in all GSM campaigns (Ziad et al. 1999). Large isolated values of $\mathcal{L}_{0}$, called "bursts", were also noted at all sites.
At Paranal we, for the first time, registered during several periods relatively small and stable outer scales. Our overall median $\mathcal{L}_{0}=22.0 \mathrm{~m}$ is smaller than at other sites studied.

In the plot of $\mathcal{L}_{0}$ given in Fig. $2 \mathrm{a}$ we note the occurrence of two nights with relatively large median values of $\mathcal{L}_{0}(45$ and $48 \mathrm{~m})$. On both these nights the wind of $6 \mathrm{~m} / \mathrm{s}$ was blowing from South, which is not typical for Paranal.

During any single night there is no obvious systematic correlation of $\mathcal{L}_{0}$ with seeing, even if a poor correlation appears sometimes.

The bursts of $\mathcal{L}_{0}$ exist at Paranal, as well as at other sites.

Curiously, a burst occurred at about the same time, 03h30 UT, during several nights. We believe that this effect is not an artefact, but reflects some real changes in the turbulence regime at Paranal site. Persistent local effects occurring in the SL at the same local time during several nights were also found (see below).

Table 1. Data summary

\begin{tabular}{cclcccc}
\hline Date & \multicolumn{2}{c}{ Wind at $10 \mathrm{~m}$} & $\begin{array}{c}\varepsilon \\
\text { " }\end{array}$ & $\begin{array}{c}\mathcal{L}_{0} \\
\mathrm{~m}\end{array}$ & $\begin{array}{c}\theta_{0} \\
\prime \text { " }\end{array}$ & $\begin{array}{c}\varepsilon_{\text {DIMM }} \\
{ }^{\prime}\end{array}$ \\
\hline 27.11 & 7.3 & $\mathrm{E} \rightarrow \mathrm{N}$ & 0.81 & 23.8 & 1.95 & 0.79 \\
28.11 & 0.6 & $\mathrm{~S} \rightarrow \mathrm{W}$ & 0.67 & 15.6 & 2.45 & 0.97 \\
29.11 & 6.9 & $\mathrm{SE}($ stable $)$ & 0.99 & 45.3 & 1.68 & 1.05 \\
30.11 & 3.6 & $\mathrm{~S} \rightarrow \mathrm{W}$ & 1.02 & 21.7 & 1.90 & 1.00 \\
1.12 & 4.1 & $\mathrm{~S} \rightarrow \mathrm{NW} \rightarrow \mathrm{NE}$ & 0.90 & 16.7 & 1.78 & 0.85 \\
2.12 & 5.4 & $\mathrm{~N} \rightarrow \mathrm{E} \rightarrow \mathrm{N}$ & 0.88 & 16.9 & 1.75 & 0.85 \\
3.12 & 4.8 & $\mathrm{E} \rightarrow \mathrm{N}$ & 0.79 & 16.8 & 1.97 & 0.76 \\
4.12 & 4.5 & $\mathrm{E} \rightarrow \mathrm{N}$ & 0.81 & 17.0 & 2.17 & 0.69 \\
5.12 & 5.2 & $\mathrm{E} \rightarrow \mathrm{N}$ & 0.90 & 16.5 & 2.09 & 0.87 \\
6.12 & 8.3 & $\mathrm{~N} \rightarrow \mathrm{NE}$ & 0.86 & 24.5 & 1.74 & 0.84 \\
7.12 & 6.5 & $\mathrm{~N} \rightarrow \mathrm{NE}$ & 1.03 & 19.4 & 1.48 & 0.95 \\
8.12 & 3.2 & $\mathrm{~S} \rightarrow \mathrm{N}$ & 0.90 & 16.9 & 1.51 & 0.80 \\
14.12 & 6.3 & $\mathrm{~S}(\mathrm{stable})$ & 0.92 & 48.4 & 1.95 & 0.95 \\
15.12 & 3.4 & $\mathrm{SE} \rightarrow \mathrm{N}$ & 0.95 & 21.5 & 2.22 & 0.80 \\
16.12 & 3.1 & $\mathrm{SE} \rightarrow \mathrm{N} \rightarrow \mathrm{NE}$ & 0.89 & 17.5 & 2.54 & 0.96 \\
17.12 & 6.5 & $\mathrm{E} \rightarrow \mathrm{N}$ & 0.89 & 23.6 & 1.41 & 0.99 \\
18.12 & 8.8 & $\mathrm{~N} \rightarrow \mathrm{NE}$ & 1.31 & 37.0 & 3.17 & 1.46 \\
19.12 & 10.8 & $\mathrm{~N}(\mathrm{stable})$ & 0.73 & 33.9 & 2.09 & 0.83 \\
20.12 & 6.5 & $\mathrm{E} \rightarrow \mathrm{N}$ & 1.29 & 23.7 & 1.41 & 1.30 \\
\hline Average & median values & 0.88 & 22.0 & 1.90 & 0.87 \\
\hline
\end{tabular}

\section{Contribution of the surface layer to the overall seeing}

\subsection{Surface layer detection from GSM and DIMM measurements}

A comparison of seeing values measured simultaneously by ESO DIMM and GSM shows that most of the time both instruments are in excellent mutual agreement. An example of such agreement is given in Fig. 3. 

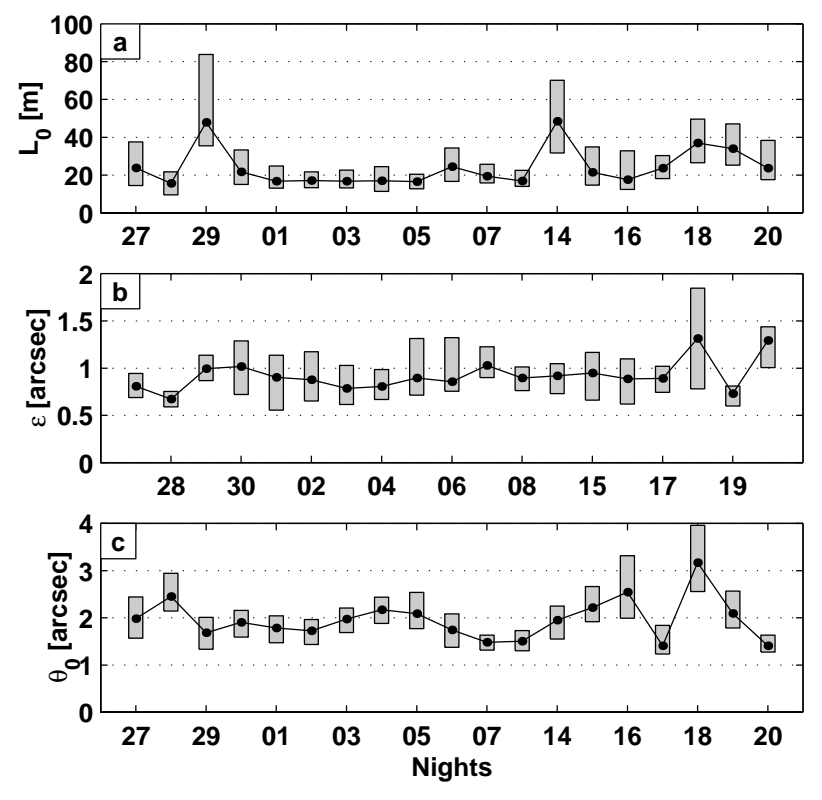
$\mathrm{L}_{0}[\mathrm{~m}]$
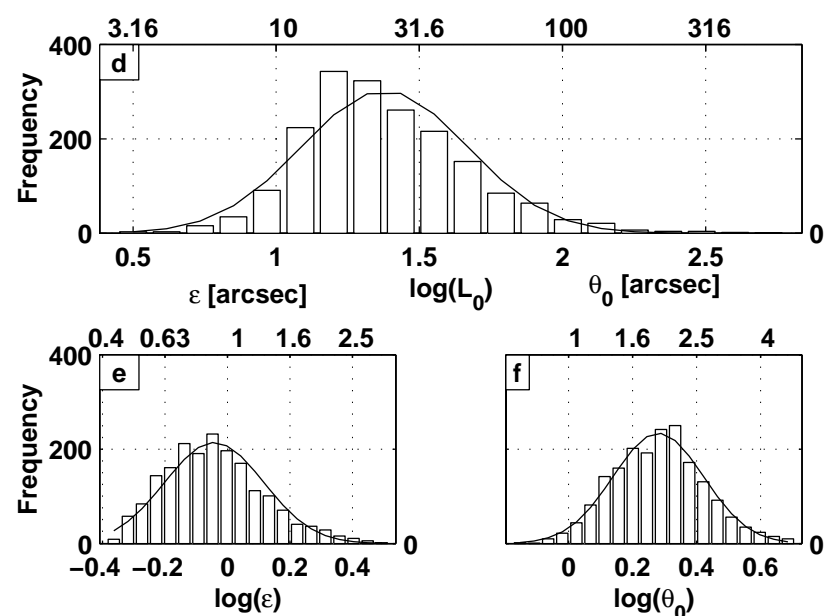

Fig. 2. Summary of the GSM data at Paranal: evolution of a) wavefront outer scale $\mathcal{L}_{0}, \mathbf{b}$ ) seeing $\varepsilon$ and $\mathbf{c}$ ) isoplanatic angle $\theta_{0}$ during the 19 nights of the mission. For each night the median value of the considered parameter is plotted as a dot and the rectangle indicates the range in which $50 \%$ of the values are included. The histograms of these 3 parameters $\mathbf{d}$ ), e) and f) correspond closely to a log-normal distribution

However, for some periods GSM measured a worse seeing than DIMM because of the lower height of GSM, which makes it more sensitive to the SL turbulence. This situation appeared soon after the beginning of our mission as an enigmatic effect: for several consecutive nights, starting from December 1 and until December 5, GSM registered a much worse seeing than DIMM for a period of about one hour, around local midnight. For the rest of the time, the agreement was good. A most clear example of this phenomenon (which we call here midnight local turbulence, or MLT) is given in Fig. 4. On December 2, starting from 03h00 UT, GSM gave a seeing systematically worse than
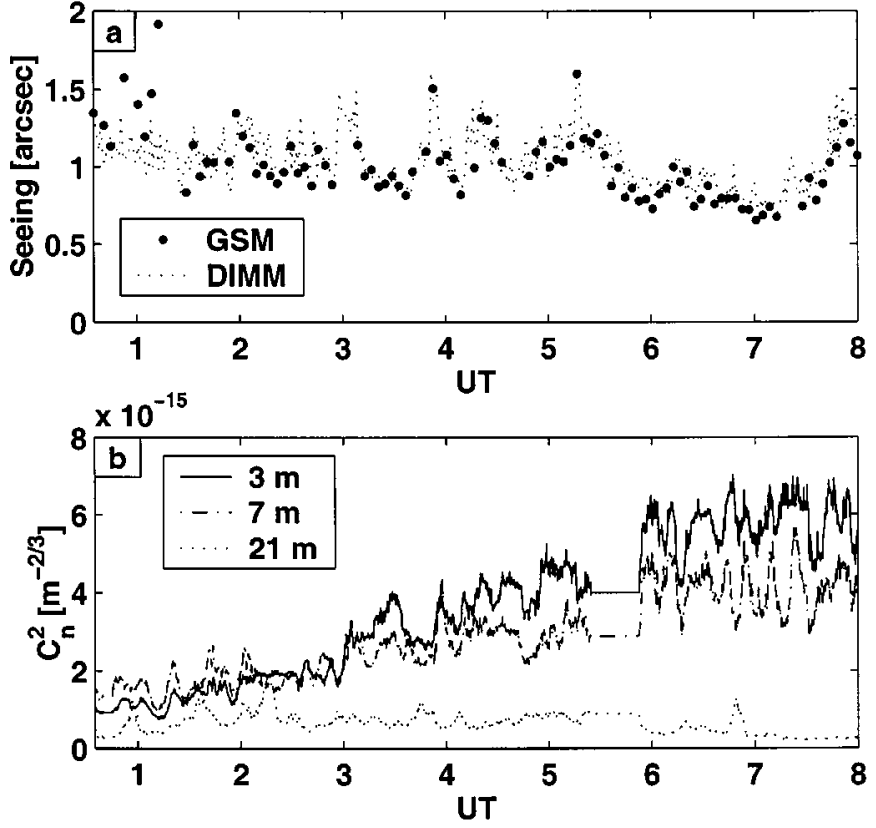

Fig. 3. The seeing measured by GSM (full circles) and ESO DIMM (dotted line) on the night of November 29 is plotted in the upper panel, showing an excellent agreement between both instruments. Simultaneous recordings of the $C_{n}^{2}$ with temperature sensors at the heights of 3, 7 and $21 \mathrm{~m}$ are plotted in the lower panel

that given by DIMM. This particular local phenomenon (MLT) is explained in the next subsection.

\subsection{Statistical contribution of the surface layer to the global seeing}

The micro-thermal data, also plotted in Figs. 3 and 4, confirm the idea that the divergence between GSM and DIMM seeing values is caused by the strong turbulence in the first few meters above the ground. Indeed, the period of disagreement exactly matches the turbulence increase at $3 \mathrm{~m}$. Similar behavior was observed on the nights of December $1-5$ corresponding to the period of stable atmospheric conditions, already noted in Sect. 3. Ground wind of $5 \mathrm{~m} / \mathrm{s}$ was prevailing during this period, with a direction slowly changing from East to North during the night, and with moderate velocity variations.

A simple statistical processing of the temperature micro-fluctuation data permits to give a global behavior of the $C_{n}^{2}$ measured at the three heights during 9 nights. For each height, about 45000 values of the structure constant have been recorded and processed. The results can be seen in Fig. 5 which shows clearly that statistically the $C_{n}^{2}$ mean value near the ground (at $3 \mathrm{~m}$ ) is greater than the one at $21 \mathrm{~m}$. The ratio of the corresponding values of $C_{n}^{2}$ is approximately 16 . Conversely, the dispersion grows with height. 


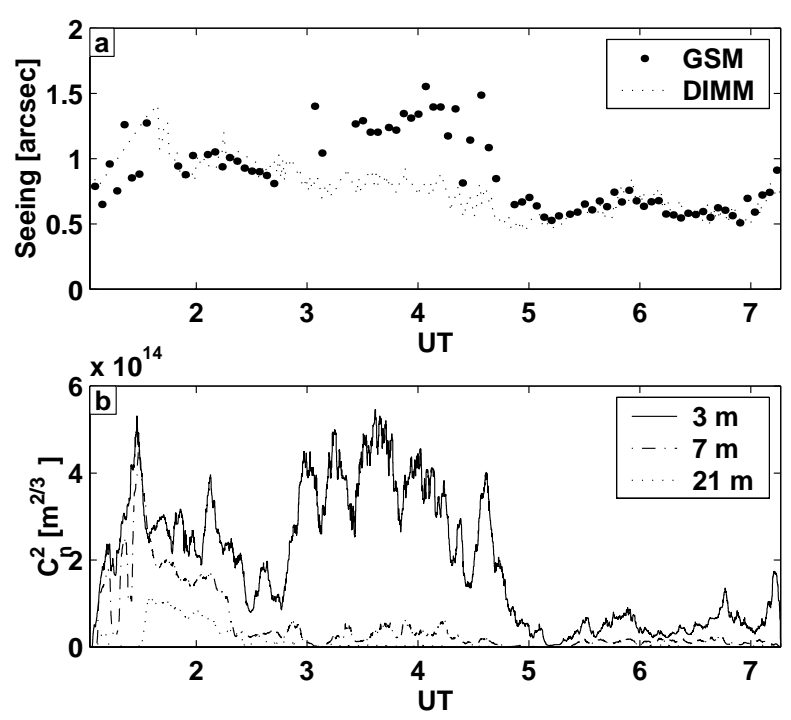

Fig. 4. Same as Fig. 3 for the night of December 2. The local turbulence around midnight caused the difference of the seeing measured by GSM and DIMM. It matches the increase of $C_{n}^{2}$ at the height of $3 \mathrm{~m}$

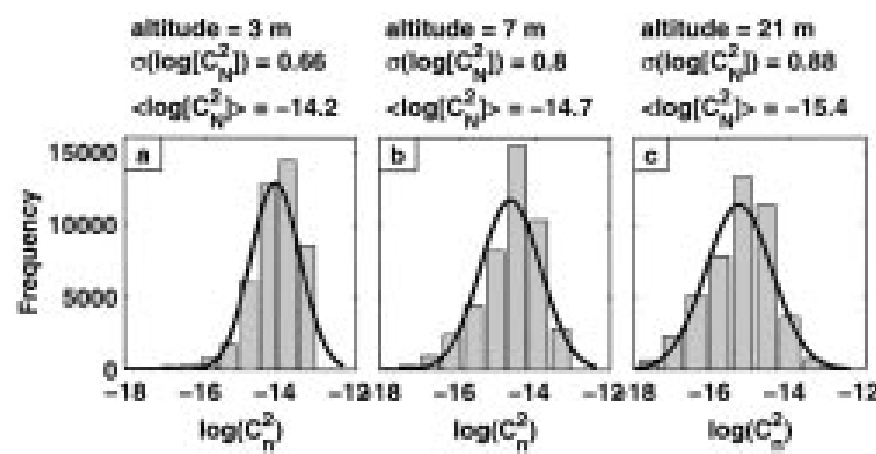

Fig. 5. Histograms of the $C_{n}^{2}$ values computed from the temperature sensors at 3 altitudes, a) $3 \mathrm{~m}$, b) $7 \mathrm{~m}$ and c) $21 \mathrm{~m}$

To estimate the relative contribution of the SL to the total seeing degradation, a turbulent energy ratio (TER) can be defined as the ratio of the turbulent energies in the $(3 \mathrm{~m}-21 \mathrm{~m})$ and $(3 \mathrm{~m}-$ infinity) layers:

$$
\text { TER }=\frac{\int_{3 \mathrm{~m}}^{21 \mathrm{~m}} C_{n}^{2} \mathrm{~d} h}{\int_{3 \mathrm{~m}}^{+\infty} C_{n}^{2} \mathrm{~d} h} .
$$

For all the nights during which temperature data where available, this ratio has been computed each four minutes in order to match the sampling rate of the GSM which provides the turbulent energy from $3 \mathrm{~m}$ to infinity. Numerator of the above expression is deduced from the integration of rough $C_{n}^{2}$ profile given by the temperature sensors between $3 \mathrm{~m}$ and $21 \mathrm{~m}$. Night by night mean values of the TER can be found in Fig. 6. From the table one can see that nightly values are in a ratio of 1 to 2.4 .

In comparison with the seeing produced by turbulence layers above $21 \mathrm{~m}$, a degradation would occur if the turbu-

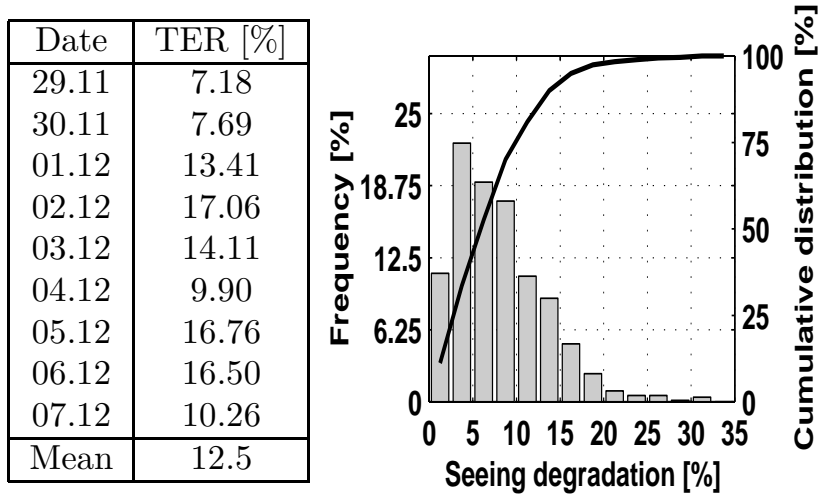

Fig. 6. Values of the turbulent energy ratio for the 9 nights for which temperature data are available. Histogram of the seeing degradation values and corresponding cumulative distribution

lent effects in the SL were added. The value of the seeing degradation $(\%)$ is given by $\left(1-[1-T E R]^{0.6}\right)$.

Histogram of the seeing degradation values and its cumulative distribution are plotted in Fig. 6. The median value is $7.2 \%$. Fifty percent of the values centered on the median are between $4.0 \%$ and $11.0 \%$.

It is thus evident that the contribution of the SL to the global seeing cannot always be considered as negligible and, although GSM is more affected by SL turbulence, DIMM is not always entirely free of it either. Ground turbulence, a part of which appears above DIMM but below VLT, may partially explain the difference sometimes detected between UT telescopes and DIMM seeings.

\section{Conclusions}

The GSM mission to Paranal was highly successful and for the first time provided statistical data on the outer scale at this site. Our main results consist in the following:

- The distribution of the outer scale $\mathcal{L}_{0}$ at Paranal is close to log-normal, with a median value of $22.0 \mathrm{~m}$. It means that because $\mathcal{L}_{0}$ is comparable to the telescope aperture size the effects of the finite outer scale on the operation of VLT are significant and result in reduction of image motion;

- The correlation of $\mathcal{L}_{0}$ with local meteorological conditions was noted. For typical conditions of NE wind, stable nightly median values of $\mathcal{L}_{0}$ around $16 \mathrm{~m}$ are found, whereas for the southern wind (associated with the wind shear in the boundary layer) they are over $45 \mathrm{~m}$;

- Excellent agreement of the seeing measured by the GSM and by the ESO DIMM is found. Median seeing for our campaign was $0.88^{\prime \prime}$ for GSM and $0.87^{\prime \prime}$ for DIMM;

- The median value of the isoplanatic angle $\theta_{0}$ was $1.90^{\prime \prime}$ during our mission. This appears as an intermediate value in comparison with our estimations during previous measurement campaigns at other observatories 
$\left(1.26^{\prime \prime}\right.$ at La Silla, 2.48" at Maidanak and $2.73^{\prime \prime}$ at Cerro Pachon). Thus, the high-altitude turbulence over Paranal appears to be not substantially different from other observatories;

- The strong local turbulence in the first few meters of the surface layer exists at Paranal for some periods of time, as evidenced by DIMM-GSM comparison and micro-thermal mast measurements;

Turbulent energy in the SL contributes an average amount of only $12.5 \%$ to the total turbulent energy detected by GSM along the line of sight. This corresponds to an average seeing degradation around $8 \%$ if turbulence effects in the SL are added to the turbulence above $21 \mathrm{~m}$. While unit telescopes of the VLT are not affected by these layers, the situation will not be the same for auxiliary telescopes (ATs) of the VLTI which have been designed closer to the ground $(4.5-6.5 \mathrm{~m})$. Hence, continuous micro-thermal monitoring at Paranal appears to be desirable to study the local effects in detail and to evaluate their importance for the site seeing and particularly for the ATs;

- The temporal evolution of atmospheric parameters at Paranal seems to be rather specific (prolonged periods of stable conditions; dependence on the wind speed and its fluctuations; repeatable increases of surface layer turbulence) compared to other sites, and warrants further investigation. A better understanding of the mechanisms of Paranal seeing will eventually lead to forecast observing conditions.

The analysis of the spatio-temporal cross-correlations of the AA fluctuations can lead to the detection of the atmospheric layers (Avila et al. 1997). From the AA crosscorrelations one can estimate the number of predominant turbulent layers, their respective strengths, the velocity and direction of the wind which carries the turbulent eddies. The principle of the wind measurement with GSM is given in Conan et al. (1998). The full wind analysis and cross-correlation interpretation must include simultaneous comparisons with other parameters, $\mathcal{L}_{0}, \varepsilon$ and $\theta_{0}$. The methods of such complete analysis are still under development.

Acknowledgements. The success of this observing campaign is a result of a long and tedious preparatory work done at the Département d'Astrophysique of the Nice University. We acknowledge the efforts of A. Robini, J.-F. Manigault and M. Azouit in the development of GSM and of the mast equipment, and the help of the staff of the "Observatoire de la Côte d'Azur". On-site preparation of GSM campaign at Paranal was organized and implemented by ESO under the supervision of M. Sarazin. We are indebted to Francisco Gomez for helping with observations during the second half of the mission.

Financial support came essentially from INSU, CNRS and MENRT for development of GSM experiment, and from ESO for this measurement campaign.

\section{References}

Avila R., et al., 1997, J. Opt. Soc. Am. A 14, 3070

Berio P., et al., 1997, J. Opt. Soc. Am. A 14, 114

Borgnino J., 1990, Appl. Opt. 29, 1863

Buscher D.F., et al., Appl. Opt. 34, 1081

Colavita M.M., et al., 1987, Appl. Opt. 26, 4106

Conan R., et al., 1998, Proc. ESO, Topical Meeting on Astronomy with Adaptive Optics, Present results and future programs, Sonthofen

Davis J., et al., 1995, MNRAS 273, L53

Fried D.L., 1982, J. Opt. Soc. Am. 72, 52

Mariotti J.M., Di Benedetto G.P., 1984, Proc. IAU Colloq. 79, 257

Martin F., et al., 1994, A\&AS 108, 173

Martin F., Tokovinin A., Ziad A., Conan R., Borgnino J., Avila R., 1998a, GSM measurement campaign at La Silla August 26th-September 20th, 1997, ESO report VLT-TRE-UNI17440-0005

Martin F., et al., 1998b, A\&A 336, L49

Rigaut F., et al., 1991, A\&A 250, 280

Roddier F., et al., 1982, J. Opt. (Paris) 13, 263

Sarazin M., 1997, Proc. SPIE 3125, 366

Sarazin M., Roddier F., 1990, A\&A 227, 294

Takato N., Yamaguchi I., 1995, J. Opt. Soc. Am. A 12, 958

Tokovinin A., Kornilov V., Conan R., Ziad A., Martin F., et al., 1998a, Report on the Maydanak site testing campaign in July 1998, INTAS-96-0367-Progress report No. 1 Tokovinin A.A., et al., 1998b, Proc. SPIE 3353, 1155

Vernin J., Muñoz-Tuñon C., 1992, A\&A 257, 811

Ziad A., et al., 1994, A\&A 282, 1021

Ziad A., et al., 1999, From Grating Scale Monitor to Generalized Seeing Monitor (submitted to Appl. Opt.) 